\title{
Impulse non-stationary flooding of hydrocarbon deposits
}

\author{
Khabibullin Marat Yakhievich \\ Ufa State Petroleum Technological University, \\ Branch of the University in the City of Oktyabrsky, Russian Federation \\ E-mail: m-hab@mail.ru
}

\begin{abstract}
Pulse method of non-stationary flooding is a continuous regulation process. Its efficiency is determined by the reserve recovery stage and is chosen after thorough analysis of the development, when the oil deposits are determined differentially on the field zones and deposits and there is a positive forecast of their extraction by existing methods. Then comparing development parameters of different variants of pulse non-stationary flooding the best development method is chosen. In this case bottom-hole or well head devices (energizers, shaker apparatus, pulsators etc.) are used for pulse flooding in order to boost reverse capillary imbibitions of closed oil zones and reversal filtration of the injected water. Experiments showed, with the increase of vibration frequency at the given parabolic law of pressure deviation fluid penetration rate into the fractured-porous layer increases sharply. Preliminary results showed high efficiency of selective application of complex flooding of hydrocarbon deposit.
\end{abstract}

Keywords -water injection well, capillar, parabolic, law, frequency.

\section{INTRODUCTION}

Efficiency of impulse non-stationary flooding as method of development regulation depends on successful choice of a rational variant with the account of certain conditions of deposits development. Practical experience of impulse nonstationary flooding (with additional application of pulsing devices down the hole or on the reinjection well head) shows that it should be used with caution especially developing new deposits. Method variants with less amount of water are suitable only for flooded deposits with mechanized water extraction.

Pulse non-stationary flooding is a continuous regulatory process. Its variant is chosen after thorough analysis of the development, when oil deposits are determined differentially in the field zones and there is a positive forecast of their extraction with the existing methods. Then comparing development parameters of different variants of impulse non stationary flooding with that one of the non-stationary flooding the best method is chosen.

\section{MATERIALS AND METHODS}

To estimate the results and efficiency of impulse stationary flooding it is necessary to compare the effects of elastic capillary imbibition of closed pores change of filtration direction during pulse non-stationary flooding. In this case bottom-hole or well head devices (energizers, shaker apparatus, pulsators etc) are used for pulse flooding in order to boost reverse capillary imbibitions of closed oil zones and reversal filtration of the injected water [1-3]. Such complex flooding was tested in 2015-2016y.y. and it proved efficient.

For the elastic capillary imbibition to be effective half cycle duration should be in the range $t=\frac{R_{K}^{2}}{4 x \cdot 0,5}$, where $\mathrm{R}_{\mathrm{c}}-$ feed contour radius, $\chi$ is formation pressure conductivity. If $\mathrm{R}_{\mathrm{c}}=500 \mathrm{~m}, \chi=10^{4} \mathrm{sm}^{2} / \mathrm{s} \quad$ (average parameters), this condition is valid at $\mathrm{t}>2$ day, that is in all oil deposits zones where it was fulfilled. But some zones had no positive effect. Let's determine effect conditions of the reverse filtration and at water injection well change of mode.

Such mode of operation will be effective when capillar imbibition is enhanced by reverse filtration which allows injecting water smoothly.

In areal development systems these conditions are fulfilled by the geometry and position of water injecting and extracting wells.

In block systems peculiarities of mutual position of water injecting and extracting wells allow many variants of operation modes (singular, in groups, in blocs etc). That is why it is necessary to take into account influence of consequent operation mode change on the final effect of pulse non-stationary flooding and the choice of the rational variant in given conditions.

Strict analytical solution on determining quality effect and pulse non-stationary flooding in this formulation is rather difficult [4]. But if we solve several practical problems, for example choose the best variant, its fulfillment; we will get comparative characteristics of process efficiency. In block development systems the criteria of relative efficiency of pulse non-stationary flooding can be the speed of filtration parallel to the pumping row. Using this parameter as a criterion is justified [5].

Lateral coning is known to go narrow from water injection wells to extracting wells. As here the pressure differential is maximal. That is why solution of the problem of a uniform oil displacement and increasing of waterless extraction comes down to injected water flow increase in the direction parallel to the water injection wells row. Injected water arrival intensity index in this direction equals to corresponding component of filtration speed. Filtration speed component variation value in any direction does not give absolute values of oil recovery, water cutting lessening and so on. It allows receiving comparative estimation of different operational 
modes of water injecting wells, when capillar imbibition is enhanced by reverse fluid direction [6].

Now, let's study the influence of viscous-capillar cyclic oil-water displacement on blocks capillar imbibition acceleration. In reality this method consists of periodic pressure change or flow rate on the layer limits resulting in periodic change of these parameters during the contact of highly porous and low permeability на objects of a layer.

During pressure increase oil in porous blocks, lenticles or interlayers is pressed and water penetrates into them. When the pressure is lowered oil and water expand but water is held by capillary forces in non-uniformities of the layer and oil exits them.

In viscous-capillar cyclic mechanism there are two effects that influence its result. The first is acceleration of water penetration into layers' non-uniformities as the result of average pressure drop increase between them. The second effect is capillar water hold in non-uniformities.

To estimate water penetration acceleration into nonuniformities let's deal with porous-fractured layer, using an equation of liquid exchange between blocks and fractures [7], after mathematical manipulations we receive equation to determine speed of liquid penetration into non-uniformities.

$$
v=\frac{1}{e^{b t}}\left\{\frac{2 \alpha}{\mu}\left[\frac{a^{2} e^{b t}}{b^{2}}(b t-1)-\frac{\sqrt{P_{\min }} a \cdot e^{b t}}{b}\right]+C\right\} .
$$

Taking into account stationarity of fluid oscillation onset that is $v(a, t)=v(t)$, specific equation solution (1) will be

$$
v=\frac{2 \alpha a}{\mu b}\left[\frac{a}{b}(b t-1)-\sqrt{P_{\min }}\right]
$$

where $a=\frac{\sqrt{P_{\max }}-\sqrt{P_{\min }}}{\varphi_{1}} \omega ; \quad b=\frac{\alpha}{\mu \beta_{2}}$,

$\alpha$ is a coefficient characterizing fluid exchange intensivity between blocks and fractures;

$$
\begin{aligned}
& \mu \text { - oil viscosity, } \mathrm{Pa} \cdot \mathrm{s} ; \\
& \omega \text { - angular frequency, } \mathrm{Rad} / \mathrm{sec} \\
& \beta_{2} \text { - blocks elasticity, } 1 / \mathrm{Pa} \text {. }
\end{aligned}
$$

Average fluid flow per cycle in one direction (e.g. from fractures to blocks), with the account of introduced designations after manipulations will be

$$
\bar{v}=\frac{2 \alpha a t_{1}}{T \mu b}\left[\frac{a}{b}\left(\frac{b t_{1}}{2}-1\right)-\sqrt{P_{\min }}\right],
$$

where $T$ is pressure change cycle, s;

$t_{l}$ is a period corresponding to pressure increase moment, $\mathrm{s}$.

Basing on the formula (2) the dependence is created $v=$ $f\left(t_{1}\right)$ at different fluid vibration frequency (see. pic.1), also dependence based on the formula (3) $v=f(\Delta P$, where
$P_{\max }=$ const), shown in picture 2 , with the following initial data: a parallelepiped is chosen as a tested formation ( $e \mathrm{x} e \mathrm{x}$ $d=20 \times 20 \times 200 \mathrm{sm}$ ), then

$$
S_{y}^{2}=\left[\frac{2(e+2 d)}{e d}\right]^{2}=\frac{4(e+2 d)^{2}}{e^{2} d^{2}}=\frac{4(20+200)^{2}}{20^{2} \cdot 100^{2}}=0,048 \frac{1}{\mathrm{sm}^{2}} ;
$$

where: $\alpha=k_{2} \cdot S_{y}{ }^{2}=10^{-10} \cdot 0,048=48 \cdot 10^{-7}$;

$$
\begin{aligned}
& \mu=10 \mathrm{sPz} ; \\
& \beta_{2}=10^{-9} 1 / \mathrm{Pa} ; \\
& P_{\text {Max }}=10 \mathrm{MPa} ; \\
& P_{\text {Min }}=1 \mathrm{MPa} \text { (for the first dependance); } \\
& T=0,1 \mathrm{~s} ; \\
& \gamma=6^{0} .
\end{aligned}
$$

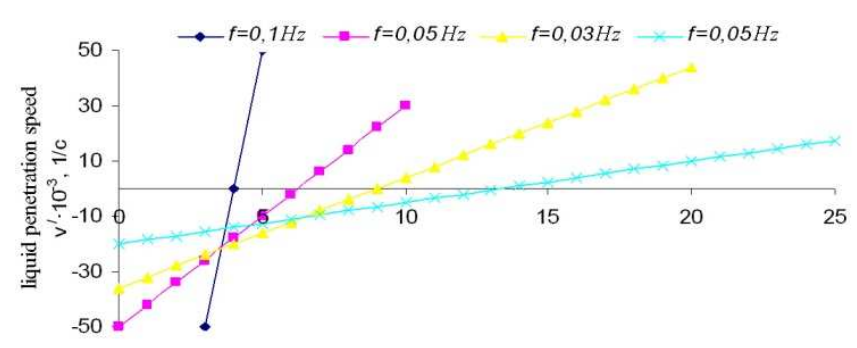

$$
\text { period corresponding to pressure increase, } \mathrm{t}_{1}, \mathrm{~s}
$$

Fig. 1. Dependence of fluid penetration speed into non-uniformities on fluid oscillation frequency

As $\bar{v}$ is fluid volume from blocks into fractures per time unit to fractured-porous layer volume unit, then total fluid volume in fractured-porous medium per volume unit will be $m$ $=m_{1}+m_{2}=0,14+0,20=0,34$. Then, on the condition that oil will be substituted by water we can determine time of fluids exchange in the given sample

$$
t=\frac{m}{v}
$$

As graphs in pictures 1 and 2 show, with oscillation frequency increase at the given parabolic law of pressure change fluid penetration speed into fractured-porous layer sharply increases. As for pressure change, efficient fluid penetration starts from values $6 \ldots 7 \mathrm{MPa}$, with oscillation frequency increase this value can be lessened.

It is important not only to "inject" the fluid into the nonuniformities, but also to hold it there by capillar forces while oil is flowing out. For quality estimation let's have a look at the model process. Let a parallelepiped porous sample be in less wettable phase. Lateral surfaces and one frontal surface are non-penetrable and a free frontal surface at the initial moment touches the fluid. Reverse capillar imbibition starts, that is wetting phase will be taken in and non-wettable will flow through the only non-wetted frontal surface. Apparently, fluid will be taken in by small pores, and non-wetting phase will flow out by big pores. 


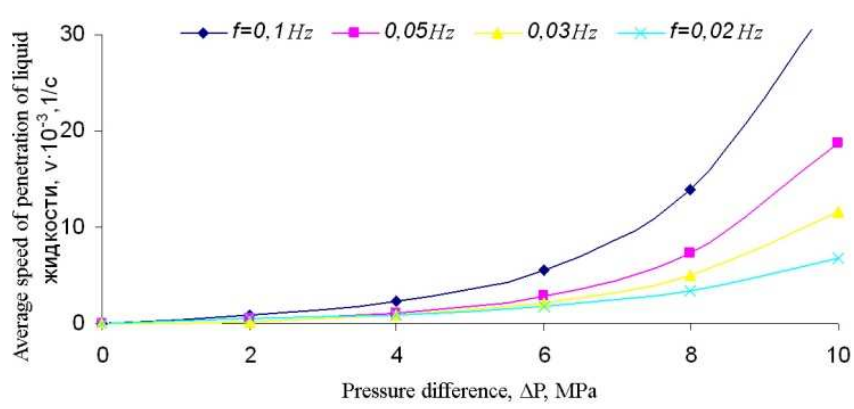

Fig. 2. Dependence of fluid penetration average speed into non-uniformities on pressure change $\Delta$

\section{RESULTS AND DISCUSSIONS}

As experiments on reverse imbibition of clear samples show [4], filtration of both phases in reverse directions is uniform during all flow; every phase takes its own pore channels system. Reverse imbibition can be studied as ordinary one-dimensional two-phase filtration. Relative penetrability for reversed flow can differ from corresponding functions during one-dimensional flow of both phases. But, in further quality study this difference is not taken into account.

Experimental studies [4] on holding water in nonuniformities during cyclic impact showed that time necessary for injected water to hold in non-uniformities completely, is approximately equal to time necessary for reversed or direct capillar imbibition of the given non-uniformity with the rest of the layer. Now, let's compare speed of oil "substitution" in a block during cyclic impact on the layer to the speed of reversed capillar imbibition, which is shown by formula [7]:

$$
\bar{\varphi}\left(t_{*}\right)=\frac{m_{2} \cdot S_{1} \cdot \sigma \cdot \cos \theta \cdot \sqrt{\frac{k_{2}}{m_{2}} \cdot S_{y}^{2}}}{\tau \cdot \mu}
$$

where $m_{2}$ is blocks porosity, 0,2 ;

$S_{l}$ is blocks water saturation, 0,5 ;

$\sigma$ is surface tension, $34,4 \cdot 10^{-3} \mathrm{H} / \mathrm{m}$;

$\cos \theta$ is wetting angle, 0,6 ;

$\tau=25,3$, is determined experimentally [7].

The value received by formula (5), should be compared with average flow per cycle by formula (3) at different fluid vibration frequency. The comparison is shown in picture 3 with the account of formation compressibility decrease in the course of time.

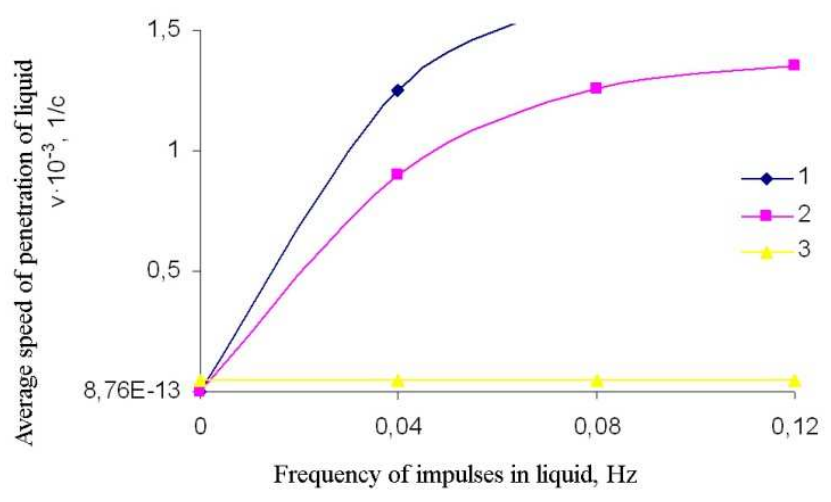

1 - theoretical speed of water penetration into non-uniformity; 2 the same with the account of formation compressibility decrease; 3 reverse capillar imbibition speed

Fig. 3. Graph of fluid penetration speed into non-uniformity and reverse capillar imbibition

The comparison shows that with injected fluid vibration frequency acceleration the speed of reverse capillar imbibition is much less than fluid penetration speed into nonuniformities. It should be born in mind that pulsation increase destroys blocks structure by mechanoactivational processes, lowering the efficiency of capillar imbibition. That is why this method will be effective if the pulsation of the injected fluid will increase gradually. We can say that greater filtration speed component parallel to the row of dividing injection wells, more uniformly oil extraction will go [7, 8].

Let's consider a part of formation with injection wells rows and exploitation rows (picture 4). Let's choose coordinate axes so that one of the axes coincides with the line of injection wells. For point $\mathrm{M}$ with coordinate $X$ and absciss $y$ of the chosen system, filtration speed components along axes are equal $V_{x} \mathbf{K} V_{y}$ correspondingly. As the direction of filtration speed component coincides with the injection wells line, the efficiency of cyclic flooding will be determined by incremental value $V_{x}$ on functional mode change of injecting wells. Filtration speed projection onto $\mathrm{X}$-axis value that is $V_{x}$ depends on injection wells functional mode change. The value of speed filtration projection onto x-axis i.e. $V_{x}$ is analytically determined by expression

$$
V_{x}=-\frac{K}{\mu} \cdot \frac{d_{p}}{d_{x}},
$$

where $K$ is layer penetrability;

$$
\begin{aligned}
& \mu \text { is fluid viscosity; } \\
& \frac{d_{p}}{d x} \text { is pressure gradient. }
\end{aligned}
$$

Pressure distribution in the point which is at distance $r$ from the source of pressure is determined by an integralexponent function [9]: 


$$
\Delta P(r, t)=\frac{Q_{\mu}}{4 \pi k h}\left[-E_{i}\left(-\frac{r^{2}}{4 x t}\right)\right]
$$

where $X$ is formation pressure conductivity.

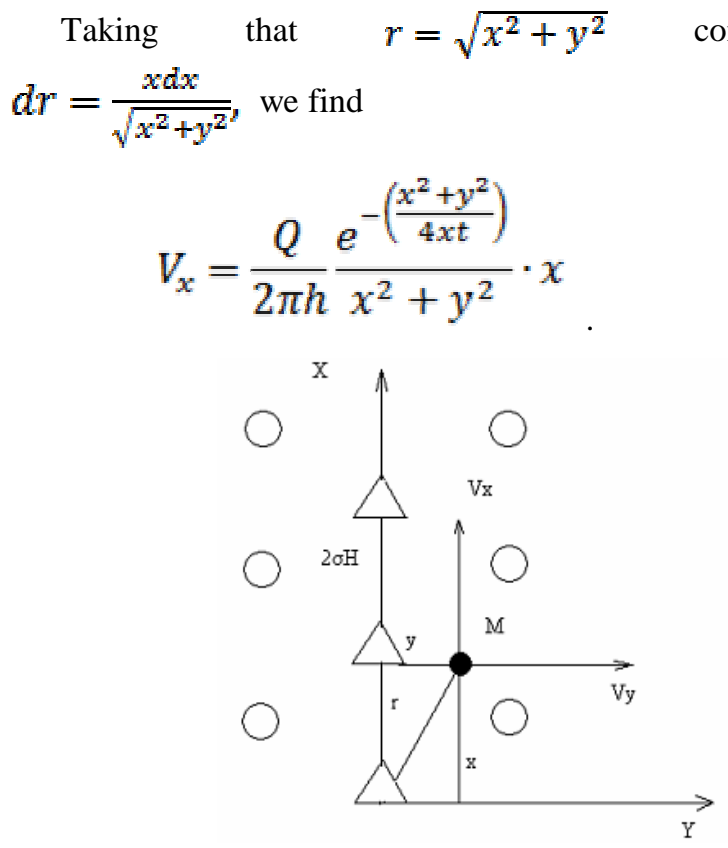

Fig. 4. Calculating scheme

For practical use it is convenient to show last formula as function of relation of $x$ value to half-distance between injection wells $\sigma_{\mathrm{H}}$ [10]. As the result we have

$$
V_{x}=\frac{Q}{2 \pi h \sigma_{\mathrm{H}}} \cdot \frac{e^{-\frac{\sigma_{\mathrm{H}}^{2}}{4 x^{t}} \cdot\left(\frac{x^{2}}{\sigma_{\mathrm{H}}^{2}}+\frac{y_{\mathrm{H}}^{2}}{\sigma_{\mathrm{H}}^{2}}\right)}}{\left(\frac{x^{2}}{\sigma_{\mathrm{H}}^{2}}+\frac{y^{2}}{\sigma_{\mathrm{H}}^{2}}\right)} \cdot \frac{x}{\sigma_{\mathrm{H}}}
$$

Let's introduce non-dimensional filtration speed change on ordinates axes [10]:

$$
V_{x}^{*}=V_{x} \frac{2 \pi h \sigma_{\mathrm{H}}}{Q}
$$

To simplify, let's take only $\mathrm{y}=0$. Calculation results of non-dimensional filtration speed changes for different values $\frac{\sigma_{\mathrm{H}}^{2}}{4 x t}, \frac{x}{\sigma_{\mathrm{H}}}$ components on axes are vectors, choosing a rational variant of non-stationary flooding it is necessary to take into account not only an absolute value of incrementation but also, direction. It follows that for two simultaneously stopped injection wells, with the distance between them, filtration speed change goes in opposite directions along the line parallel to injection wells row; that is an absolute value of the final speed change is determined by the difference of absolute values of speed change from every well. In the rest part of the layer, in relation to the denoted strip, it is determined vice-versa, by addition. As an illustration to estimate the influence of mode change order on the efficiency of filtration direction change let's take the part of the injection wells row for wells, with constant intake capacity (fig. 6).
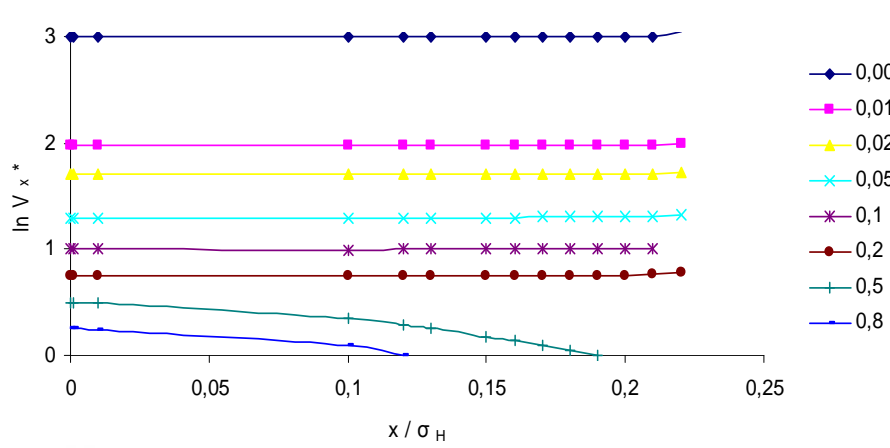

$-0,01$

$\therefore-0,02$

$-0,05$

$* 0,1$

$\rightarrow 0,2$

$+0,5$

$-0,8$

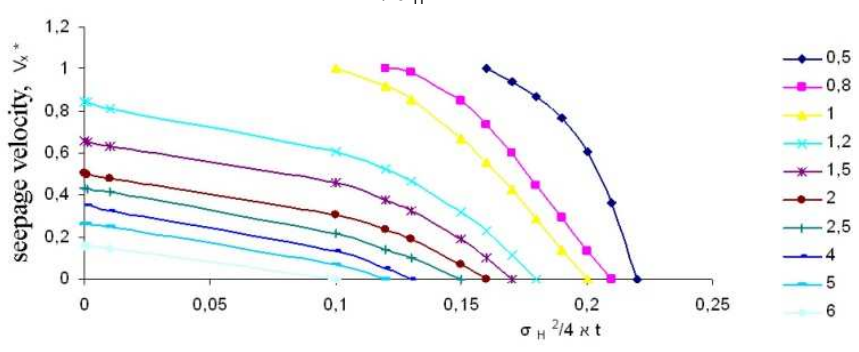

Fig. 5. Value dependence $V_{x}^{*} \frac{\sigma_{\mathrm{H}}^{2}}{4 x t}$ at relation $\frac{x}{\sigma_{\mathrm{H}}}$

Picture 6 shows change curves of dimensionless filtration speed along the line of injection wells, calculated by formula (10) $[11,12]$ at $\frac{\sigma_{\mathrm{H}}^{2}}{4 x t}=0,001 ; 0,1$ is for four variant of stop: 1 consequently one by one; 2 all simultaneously; 3 well 1 together with well 3, then well 2 together with well 4; 4 well 1 together with well 4 then well 2 together with well 3.

As we can see the most effective filtration speed change along the injection well line, hence, efficiency of pulse nonstationary flooding is at consequent well stop, then the third, the fourth, the second variants go.

It is important to note that when wells are stopped simultaneously next but one, zones between them prone to stagnation and difficult for development are actively developed in this case. If neighboring injection wells change functional mode synchronously, stagnation zones can appear. Formula analysis (9) show that in block systems of development range consequence of variants according to the value of achieved filtration direction change is the same for common case $\mathrm{y}=0$, also for non-uniform and flooded layer as not its parameters but direction change in of fluid flow from every well determines the final effect. 
$\sigma_{\mathrm{H}}$ (rounded to the less value) this maximal distance will be

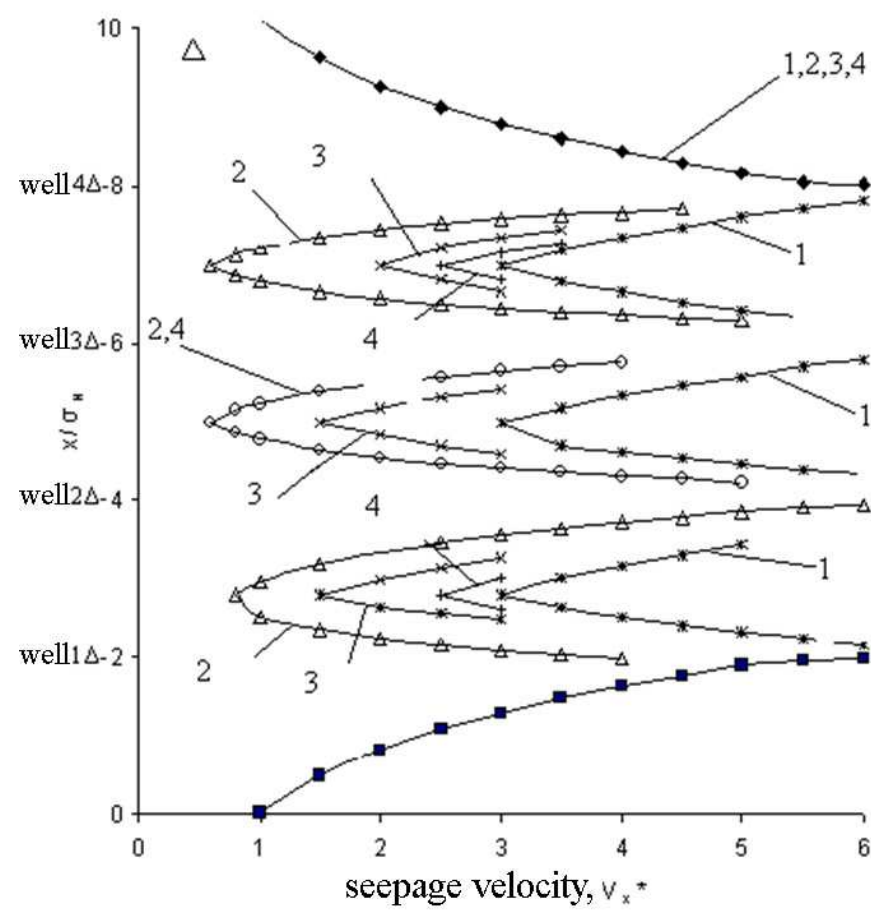
determined as relation of whole cycle duration to the less of half-cycles duration. If the half-cycles duration is equal, this relation is equal to two, that is wells in the row are stopped next but one.

But it does not exclude other variants of flooding. Conditions of deposits development, oil-water boundary advance can require significant change in filtration direction at one development zone and minor change at the other one. In this case consequence of well operational mode change should take into account the aim of the mode change. In particular, for layer zone, which is outer one to the string between injection wells, which are stopped simultaneously, filtration direction changes from every well are added. That is why, for all layer points, when extreme injection wells stopped simultaneously; there may be created more powerful instant change of filtration direction by the stop of all nearest wells, then during the stop of next but ones.

\section{References}

[1] M.Ya. Habibullin, D.I. Sidorkin, Determination of tubing string vibration parameters under pulsed injection of fluids into the well, SOCAR Proceedings, Vol. 3, pp. 27-32, 2016. https://doi.org/10.5510/ogp20160300285

[2] M.Ya. Khabibullin, R.I. Suleimanov, D.I. Sidorkin, I.G. Arslanov, Parameters of damping of vibrations of tubing string in the operation of bottomhole pulse devices, Chemical and Petroleum Engineering, Vol. 53, № 5-6, pp. 378-384, 2017. https://doi.org/10.1007/s10556-017$\underline{0350-6}$

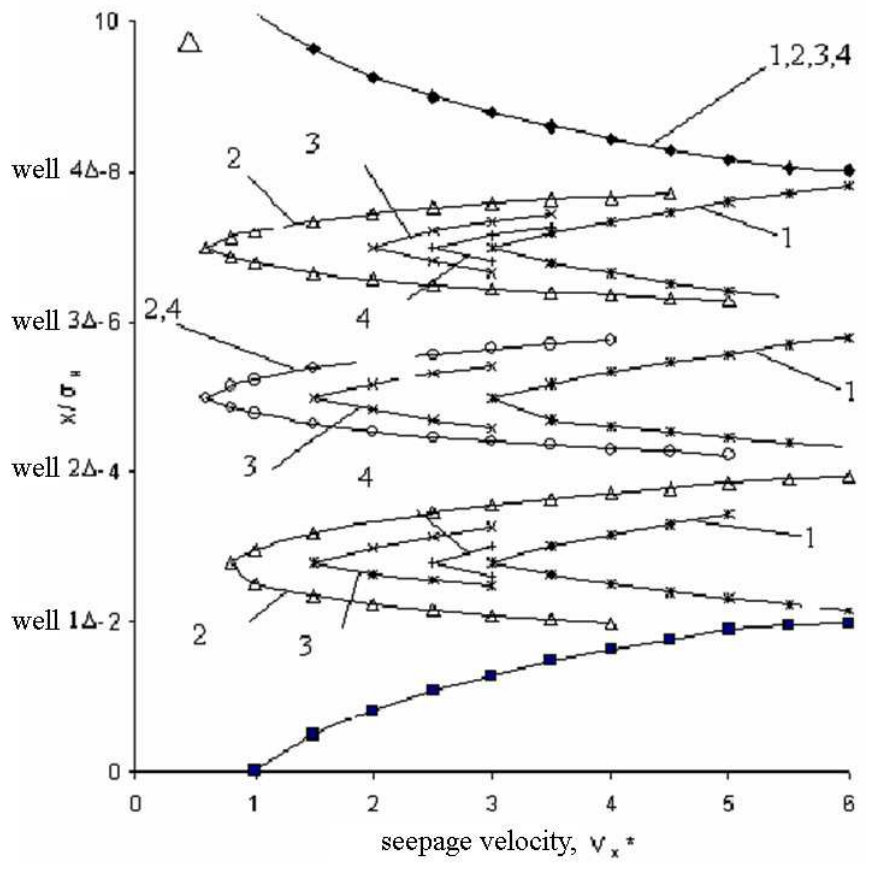

Fig. 6. Change curves $\boldsymbol{V}_{\boldsymbol{x}}^{*}$ correspondingly at 1,2,3,4 variants of injection wells stop.

$$
\mathrm{a}-\frac{\sigma_{\mathrm{H}}^{2}}{4 x t}=0,001 ; 6-\frac{\sigma_{\mathrm{h}}^{2}}{4 x t}=0,1
$$

\section{CONCLUSION}

Maximal distance between injection wells, whose operational mode can change simultaneously, is restricted by stop duration and further water injection. In fractions from 2
[3] G. A. Korn, T. M. Korn, Mathematical Handbook for Scientists and Engineers: Definitions, Theorems, and Formulas for Reference and Review. Moscow: Nauka, 1984.

[4] A. Davletbaev, V. Kireev, L. Kovaleva, A. Zainullin, R. Minnigalimov, Cold heavy oil production and production by radio-frequency electromagnetic radiation: Comparative numerical study, AIP Conference Proceedings: International Conference of Computational Methods in Sciences and Engineering 2016, ICCMSE 2016 (Athens, 17-20 March 2016) / the team of authors. - Athens: American Institute of Physics Inc., Vol. 1790, pp. 150021, 2016. DOI: 10.1063/1.4968760.

[5] M.V. Goryunova, L.S. Kuleshova and A.I. Khakimova, "Application of signal analysis for diagnostics," International Conference on Industrial Engineering, Applications and Manufacturing (ICIEAM) (Saint Petersburg: IEEE), pp. 1-5, 2017. DOI: 10.1109/ICIEAM.2017.8076487.

[6] Sun Wenyue, Hui Mun-Hong, Louis J. Durlofsky, Production forecasting and uncertainty quantification for naturally fractured reservoirs using a new data-space inversion procedure, 15th European Conference on the Mathematics of Oil Recovery (ECMOR) (Amsterdam,Netherlands), European Assoc Geoscientists \& Engineers Computational geosciences, Vol. 21, Issue: 5-6, pp. 14431458, 2017.

[7] R.M. Shaidullina, A.F. Amirov, V.S. Muhametshin, and K.T. Tyncherov, "Designing economic socialization system in the educational process of technological university," European Journal of Contemporary Education, Vol. 6 (1), pp. 149-158, 2017. DOI: 10.13187/ejced.2017.1.1494.

[8] Hourfar Farzad, Moshiri Behzad, Salahshoor Karim, Real-time management of the waterflooding process using proxy reservoir modeling and data fusion theory, Computers \& chemical engineering, Vol. 106, pp. 339-354, 2017.

[9] Wang Dashun, Niu Di, Li Huazhou, Andy Predicting Waterflooding Performance in Low-Permeability Reservoirs With Linear Dynamical Systems, SPE Journal, Vol. 22, Issue 5, pp. 1596-1608, 2017.

[10] Wei Bing, Lu Laiming, Li Qinzhi, Mechanistic Study of Oil/Brine/Solid Interfacial Behaviors during Low-Salinity 
Waterflooding Using Visual and Quantitative Methods, ENERGY \& FUELS, Vol. 31, Issue 6, pp. 6615-6624, 2017.

[11] V.V. Mukhametshin and R.R. Kadyrov, "Influence of nanoadditives on mechanical and isolating properties of cement-based compositions," (in Russian), Nanotechnologies in Construction, vol. 9, no. 6, 2017, pp. 1836. DOI: 10.15828/2075-8545-2017-9-6-18-36.

[12] Zhang Haoran, Liang Yongtu, Zhou Xingyuan, Sensitivity analysis and optimal operation control for large-scale waterflooding pipeline network of oilfield, Journal of petroleum science and engineering, Vol. 154, pp. 38-48, 2017. 